\title{
Exhausted gas reservoirs drive massive galaxy quenching in the early universe
}

Katherine Whitaker ( $\square$ kwhitaker@astro.umass.edu )

University of Massachusetts

Christina Williams

University of Arizona

Lamiya Mowla

University of Toronto

Justin Spilker

University of Texas Austin

Sune Toft

University ofCopenhagen

Desika Narayanan

University of Florida

Alexandra Pope

U Mass

Georgios Magdis

University ofCopenhagen

Pieter van Dokkum

Yale University

Mohammad Akhshik

University of Connecticut

Rachel Bezanson

University of Pittsburgh

Gabriel Brammer

University of Copenhagen https://orcid.org/0000-0003-2680-005X

Joel Leja

The Pennsylvania State University

Allison Man

University of Toronto

Erica Nelson

University of Colorado Boulder

Johan Richard

Universite Claude Bernard Lyon 1

Camilla Pacifici 
Space Telescope Science Institute

\section{Keren Sharon}

University of Michigan

Francesco Valentino

DARK, Copenhagen

\section{Physical Sciences - Article}

\section{Keywords:}

Posted Date: November 30th, 2020

DOl: https://doi.org/10.21203/rs.3.rs-111399/v1

License: (c) (7) This work is licensed under a Creative Commons Attribution 4.0 International License. Read Full License

Version of Record: A version of this preprint was published at Nature on September 22nd, 2021. See the published version at https://doi.org/10.1038/s41586-021-03806-7. 


\section{Abstract}

When the Universe was merely three billion years old, about half of massive galaxies had already formed the bulk of their stars and new star formation plummeted [1]. How galaxies quench at such early times remains a puzzle, as their dark matter halos contain large gas reservoirs [2-4]. This gas should cool efficiently, sustaining star formation over long periods $[5,6]$. Here we present sensitive $1.3 \mathrm{~mm}$ wavelength observations of cold dust in six quenched galaxies in the redshift range $z=1.6$ to $z=3.2$ with stellar masses ranging from $2.5 \times 1010 \mathrm{M} \odot$ to $5 \times 1011 \mathrm{M} \odot$, which are magnified by foreground galaxy clusters. Even with factors of up to 30 in magnification, four of the six galaxies are undetected at this wavelength. We show that these quenched galaxies have extremely little dust at early times, and by proxy very little cold molecular gas. The median dust mass is $<0.01 \%$ of the stellar mass (molecular gas mass $<1 \%$ ), more than two orders of magnitude less than star-forming galaxies at this epoch [4]. The implication is that most early galaxies shut off star formation because their reservoir of molecular gas was rapidly depleted or removed, and is not being replenished.

\section{Main Text}

The $1.3 \mathrm{~mm}$ observations were made with the Atacama Large Millimeter/submillimeter Array (ALMA) and the sample comprises of six galaxies selected from the REsolving QUIEscent Magnified (REQUIEM) galaxy survey: MRG-M13417 ${ }^{7}$ MRG-M0138 ${ }^{8}$, MRG-M2129 ${ }^{9}$, MRG-M0150 ${ }^{8}$, MRG-M0454 ${ }^{10}$, and MRG$\mathrm{M} 1423^{10}$ (Figure 1). The targets are all strongly lensed, with magnification factors ranging from a factor of 2.7 (MRG-M1423) to 30 (MRG-M1341). Five out of the six galaxies are classified as quiescent due to unusually low star-formation rates that reach down to $0.2 \mathrm{M}_{\odot} \mathrm{yr}^{-1}$, as measured from fitting the optical to infrared spectral energy distributions (see Methods). While the most distant target, MRG-M1423, has a more typical star-formation rate of $70 \mathrm{M}_{\odot} \mathrm{yr}^{-1}$ over the previous $100 \mathrm{Myr}$, consistent with normal starforming galaxies at $z=3$, its spectrum reveals classic post-starburst signatures that support a picture where it has quenched rapidly within the last $100 \mathrm{Myr}^{11}$. These targets are qualitatively different than existing millimeter/CO data tracing cold interstellar medium phases in that they have order of magnitude lower star-formation rates for their stellar mass ${ }^{11-14}$, higher redshifts ${ }^{15,16}$, and have uniquely deep flux limits facilitated by strong lensing magnification.

For the redshift range of our sample, our $1.3 \mathrm{~mm}$ wavelength observations correspond to $300-500 \mu \mathrm{m}$ restframe on the Rayleigh-Jeans tail of the dust emission, which serves as a robust proxy for the cold molecular gas mass ${ }^{17}$. We clearly detect two of the sources in the dust continuum: MRG-M0138 at $0.27 \pm 0.03 \mathrm{mJy}$ and MRG-M2129 at $9.74 \pm 0.16 \mathrm{mJy}$. With percent-level molecular gas fractions, these are the first direct detections of cold dust in individual quiescent galaxies outside the local universe. In contrast with the extended stellar light profiles, and despite the enhanced resolution from strong lensing magnification, both sources remain unresolved. This suggests that they have high dust and molecular gas surface densities, as the dust continuum is centrally concentrated and significantly less extended than the stellar light (see Figure 1). Such a result has also been found in star-forming galaxies at similar 
redshifts ${ }^{18}$. The sensitive $A L M A$ dust continuum imaging of the remaining four sources all yield strong upper limits, with the $3 \sigma$ detection limits ranging from 30-150 $\mu \mathrm{Jy}$ before lensing corrections. We estimate the dust mass, $M_{\text {dust }}$, by adopting a modified blackbody fit and making standard assumptions about dust temperature and emissivity (see Methods).

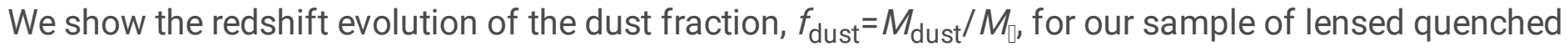
galaxies in Figure 2. By adopting a ratio of the molecular gas mass to dust mass of 100 (see Methods), we estimate $M_{\mathrm{H} 2}$ directly from $M_{\text {dust }}$ and also show the inferred molecular gas fraction, $f_{\mathrm{H} 2}=M_{\mathrm{H} 2} / M_{\square}$ (right axis). Even if we adopt an extremely conservative molecular gas to dust mass ratio that is a factor of ten higher, $f_{\mathrm{H} 2}$ is still well below that of normal star-forming galaxies at this epoch ${ }^{2}$. Both of our unambiguously detected galaxies have low molecular gas fractions of $4.6 \pm 0.5 \%$ and $0.6 \pm 0.1 \%$, respectively. Strong upper limits from $\mathrm{CO}$ emission for these two targets (A. Man et al. in prep) rule out more exotic molecular gas-to-dust ratios in these particular cases, which would otherwise imply larger cold gas reservoirs. While studies of cold gas in quiescent galaxies in the local Universe are consistent with scaling relations ${ }^{19}$, our observations reveal a population of massive galaxies at $z>1.5$ that have molecular gas fractions more than order of magnitude lower than empirical predictions. Our measured $f_{\mathrm{H} 2}$ is $0.8 \pm 0.4$ dex lower on average than scaling relation predictions for the given star-formation rates and stellar mass ${ }^{2}$.

Our program is the first to measure a broad range of (low) molecular gas masses in massive galaxies with suppressed star-formation rates (Figure 3). A comprehensive literature search at $1.5<z<3.0$ (see Methods) demonstrates that galaxies typically form copious new stars (median $\log \left(S F R / M_{0}\right)=-8.6$ ) and have a bountiful fuel supply, with a median value of $f_{\mathrm{H} 2}=51 \%$. By comparison, our galaxies instead form two orders of magnitude fewer new stars (median $\log \left(\mathrm{SFR} / M_{\mathbb{1}}\right)=-10.7$ ) and have a median upper limit of $f_{\mathrm{H} 2}<1 \%$. Until now, such low molecular gas fractions have only been measurable in galaxies in the local universe ${ }^{19}$. Our new measurements confirm that the cold interstellar medium was already rapidly depleted at high redshift in at least some galaxies, not slowly consumed until the present day. Another study has already set the stage at high redshift, finding moderate cold gas reservoirs based on stacking dust continuum measurements in a mass-representative sample, albeit at significantly lower resolution ${ }^{12}$. While the cold gas reservoir of MRG-M2129 is consistent with these first results, all other sources remain in significant tension. Our results also contradict the moderate cold gas reservoirs detected in recentlyquenched galaxies at lower redshifts that instead imply reduced star formation efficiency ${ }^{15}$. While in principle, differences in the ages of the stellar populations could explain this discrepancy ${ }^{20}$, our sample includes both recently-quenched ( 100-800 Myr) and older passively evolving galaxies ( 1.3-1.6 Gyr) ${ }^{7-10}$. The scatter in molecular gas fractions at low star-formation rates suggests a diverse range of evolutionary pathways to quiescence. The emerging picture at the highest redshifts is one where most massive quiescent galaxies either rapidly deplete their cold gas within the first few billion years of the Big Bang, or eject it into the surrounding intergalactic medium. 
Quiescent galaxies are spectroscopically confirmed as early as $z=4^{21}$. The existence of these early quiescent galaxies and the rapid and complete exhaustion of gas implied by our data are critical constraints on models of galaxy evolution, which currently struggle to produce realistic quiescent galaxies across redshift ${ }^{21}$. Predictions from cosmological simulations for the molecular gas leftover after star formation ceases span multiple orders of magnitude ${ }^{22,23}$. The essential problem is that high redshift dark matter halos contain enormous gas reservoirs ${ }^{2-4}$ that should cool efficiently and maintain steady star formation over long timescales ${ }^{5,6}$. Indeed, many early massive galaxies do just that, having star-formation rates of order $100 \mathrm{M}_{\odot} \mathrm{yr}^{-1} 24$ and sizable molecular gas reservoirs ${ }^{3}$. Our new observations show that the cessation of star formation, when it occurs, is not caused by a sudden inefficiency in the conversion of cold gas to stars but due to the depletion or removal of these reservoirs.

This lack of cold gas appears to be permanent. In the absence of a heating mechanism, the hot gas biding time in the halo of massive galaxies should theoretically cool and fall back onto galaxies within a billion years ${ }^{25}$. Yet, we do not frequently observe rejuvenation in massive galaxies ${ }^{26}$. In light of this, our observations suggest there must be a physical mechanism that effectively blocks the replenishment of the cold gas reservoirs ${ }^{27}$. In the local Universe, centrally driven winds observed in quiescent galaxies are known to clear the gas out of the system, and the central low-level active supermassive black hole has sufficient mechanical energy to heat the gas and suppress star formation ${ }^{28}$. Tentative evidence also exists at high redshifts for maintenance mode energy injection from central supermassive black holes ${ }^{29}$. This process may explain why quiescent galaxies are unable to effectively re-accrete cold gas in the subsequent 10 billion years of evolution to the present day, although there are other possibilities ${ }^{30}$. Our new data demonstrate a lack of dust, and by inference cold gas, indicating that such a physical process may have already occurred at significantly earlier times.

This study marks the first detection of the dust continuum of individual massive quiescent galaxies above $z=0$, with measurements implying low $f_{\mathrm{H} 2}$ of a few percent or less. However, the use of the dust continuum as a proxy for the interstellar medium in massive galaxies with star-formation rates must be further investigated. In particular, while securing detections of both $\mathrm{CO}$ emission and dust continuum for the same high redshift quiescent galaxy is paramount, such observations are costly with our current generation of telescopes without the help of strong gravitational lensing magnification. Upcoming upgrades to existing facilities will offer pathways forward, including the wide-area, sensitive measurements enabled by the TolTEC instrument on the Large Millimeter Telescope and the improved spectroscopic capabilities forthcoming for the next generation Very Large Array. In the meantime, this program offers valuable information to constrain the wide range of theoretical predictions.

\section{Methods}

Cosmology and Initial Mass Function Assumptions. Throughout this paper we assume a simplified cosmology of $\Omega_{\mathrm{M}}=0.3, \Omega_{\Lambda}=0.7$, and $\mathrm{H}_{0}=70 \mathrm{~km} \mathrm{~s}^{-1} \mathrm{Mpc}^{-1}$, when calculating physical parameters. Such values are commonly assumed to make literature comparisons easier, as the precise measured values 
evolve over time. We adopt the Chabrier ${ }^{31}$ initial mass function throughout, correcting literature values where appropriate.

Hubble and Spitzer Space Telescope Observations. The full details of the data reduction of the REQUIEM Hubble Space Telescope (HST) and Spitzer Space Telescope data are found in the REQUIEM methodology paper ${ }^{32}$. All targets have a minimum of 5 (up to 16) HST and 2 Spitzer/IRAC filters, covering $\lambda_{\text {rest }} \sim 1000 \AA$ to $\sim 1 \mu \mathrm{m}$. In addition to ground-based spectroscopic campaigns ${ }^{3}, H S T /$ WFC3 6141 grism spectroscopy exists for five out of the six targets, excluding MRG-M1423.

Star-formation Rate and Stellar Mass Estimates. Star formation rates and stellar mass estimates are derived from a joint analysis of photometry and ground-based spectroscopy, modeling the rest-frame ultraviolet to near-infrared spectral energy distribution ${ }^{8,10}$. These papers adopt the Calzetti ${ }^{33}$ dust attenuation curve and parameterized star formation histories when fitting the stellar continuum with stellar population synthesis models. Both exponentially decaying ${ }^{8}$ and similar star formation histories that allow linear growth before the exponential decay ${ }^{10}$ yield consistent stellar mass and star-formation rate estimates and are generally well-suited to describe quiescent galaxies ${ }^{34}$. The procedures to fit the data to stellar population models marginalize over the redshift, velocity dispersion, age, metallicity, dust attenuation, and the emission line parameters, including an analysis of systematic uncertainties introduced by the model assumptions.

Lens Model Assumptions. The full details of the lens models for all strong lensed sources presented herein can be found in the original discovery papers ${ }^{7-10}$. The magnification factor was used to correct the stellar masses and star-formation rates. However, because the dust and molecular gas fractions and the specific star-formation rates, the main focus of this paper, are relative quantities, they are independent of the details of the lens models.

Reduction of ALMA Data. ALMA 1.3mm continuum observations were carried out in programs 2018.1.00276.S and 2019.1.0027.S. The observations were designed to reach limits on $f_{\mathrm{H} 2} \sim 1 \%$; due to the range in redshift and lensing magnification within the sample, the observations reach $1 \sigma$ depths of 9$56 \mu \mathrm{Jy}$. The correlator was configured for standard Band 6 continuum observations, with $7.5 \mathrm{GHz}$ total usable bandwidth. The data were reduced using the standard ALMA pipeline and imaged with natural weighting to maximize sensitivity. The observations were designed to avoid spatially resolving the target sources to the extent possible, and reach spatial resolutions $\sim 1.0-1.5$ arcseconds. We also created lowerresolution images of each source with a $u v$ taper and found no evidence for extended emission in any source. Flux densities for the two detected sources were measured from the peak pixel values in the images. For the remaining undetected sources, we place upper limits on the $1.3 \mathrm{~mm}$ emission using the image root-mean-square values.

For the four undetected REQUIEM-ALMA galaxies, each non-detection map is divided by the magnification and the individual maps' demagnified root-mean-square defines the weight when averaging to generate a weighted stack. This methodology is similar to others in the literature for unresolved sources ${ }^{35}$, with our 
sample having roughly similar beam sizes that span 1.4-1.6 x 1.1-1.2 arcseconds. The same weights are used to calculate the average stellar mass and consequently the limit in $f_{\text {dust }}=M_{\text {dust }} / M_{\square}$ for the stack. The resulting deep $3 \sigma$ limit in the dust continuum from the undetected REQUIEM-ALMA sources is $0.81 \mu \mathrm{Jy}$ at an average redshift of $z=2.59$. For an average stellar mass of $\log _{10}\left(M_{\mathbb{}} / M_{\odot}\right)$ of 10.52 , this corresponds to $f_{\text {dust }}$ of $2.6 \times 10^{-5}$.

Molecular Gas Mass Estimates. By probing the Rayleigh-Jeans tail at $\lambda_{\text {rest }}>250 \mu m$, the dust continuum can be used as a proxy for the mass of the molecular interstellar medium, $M_{\mathrm{H} 2}$. We estimate dust mass, $M_{\text {dust }}$, from a modified blackbody fit ${ }^{36}$, assuming a dust temperature of $25 \mathrm{~K}$, a dust emissivity index, $\beta$, of 1.8 , and a dust mass opacity coefficient, $\mathrm{K}_{345 \mathrm{GHz}}$ of $0.0484 \mathrm{~m}^{2} / \mathrm{kg}^{37}$. By assuming a molecular gas to dust mass ratio, $\delta$, of $100^{37}$, we can thereby infer $M_{\mathrm{H} 2}$ from $M_{\text {dust }}$. In principle $M_{\text {dust }}$ could trace both neutral and molecular hydrogen, and quiescent galaxies at $z \sim 0$ are known to harbor non-negligible neutral gas reservoirs ${ }^{38}$. Local studies show that the neutral hydrogen contribution varies widely ${ }^{19}, 39$. While we assume that all of the hydrogen gas is in the molecular form, a significant contribution from neutral hydrogen to our dust detection would only serve to strengthen our conclusion. For comparison, we also calculate $M_{\mathrm{H} 2}$ explicitly following an empirical calibration ${ }^{17}$, finding an offset of 0.1 dex lower in $M_{\mathrm{H} 2}$, yielding even lower inferred molecular gas fractions.

An alternative viable explanation of the null detections is that $\delta$ increases dramatically for a significant fraction of early quiescent galaxies. There exists theoretical ${ }^{40}$ and observational ${ }^{41}$ evidence that in certain circumstances thermal sputtering by hot electrons could in principle efficiently destroy dust in dead galaxies. CO observations are required to rule out extreme molecular gas to dust ratios that would be necessary to reconcile our observations with higher, more typical values of $f_{\mathrm{H} 2}$. While CO observations of quiescent galaxies at $z>1.5$ are scant, such ratios are difficult to justify, as they imply that $\mathrm{CO}$ should be detectable ${ }^{42}$. At least in the case of our two detections, such exotic ratios are already ruled out by strong CO upper limits ${ }^{43}$.

We adopt a dust temperature of $25 \mathrm{~K}$, which corresponds to a luminosity-weighted temperature of roughly $30 \mathrm{~K}$. However, the cold interstellar medium of local quiescent galaxies is generally colder, with luminosityweighted dust temperatures observed to be $23.9 \pm 0.8 \mathrm{~K}$ (with a range from $17 \mathrm{~K}$ to $32 \mathrm{~K}$ ) ${ }^{44}$. While adopting significantly colder dust templates would increase our estimates of molecular gas fraction ${ }^{45}$, our upper limits would still leave room for tension. Moreover, star formation in quiescent galaxies at high redshift is generally less suppressed in comparison to local dead galaxies, and as such the expected dust temperature of the cold interstellar medium remains unclear.

Literature Comparisons. We include two additional quiescent targets at similar high redshifts of $z>1.5$ with upper limits from dust continuum measurements ${ }^{14}$ and CO measurements ${ }^{15}$., as well as results from stacking dust continuum ${ }^{12}$. For the dust continuum measurements, all data is recalibrated using the same set of assumptions applied herein. We further assemble measurements of 183 star-forming 
galaxies at $1.5<z<3.0$ from the literature, tracing molecular gas via dust continuum $14,17,46,47$ and $\mathrm{CO}{ }^{48-}$ 55 , comprising the contours presented in Figure 3.

Methods References. (see References 31-55)

\section{Declarations}

Acknowledgements: This paper makes use of the following ALMA data: ADS/JAO.ALMA \#2018.1.00276.S, ADS/JAO.ALMA \#2019.1.00227.S. ALMA is a partnership of ESO (representing its member states), NSF (USA) and NINS (Japan), together with NRC (Canada), MOST and ASIAA (Taiwan), and KASI (Republic of Korea), in cooperation with the Republic of Chile. The Joint ALMA Observatory is operated by ESO, AUI/NRAO and NAOJ. The National Radio Astronomy Observatory is a facility of the National Science Foundation operated under cooperative agreement by Associated Universities, Inc. This work uses observations with the NASA/ESA Hubble Space Telescope, obtained at the Space Telescope Science Institute, which is operated by the Association of Universities for Research in Astronomy, Inc., under NASA contract NAS 5-26555. KEW wishes to acknowledge funding from the Alfred P. Sloan Foundation, HST-G0-14622, HST-G0-15663, and the unwavering support of her partner amid the global pandemic. CCW acknowledges support from the National Science Foundation Astronomy and Astrophysics Fellowship grant AST-1701546 and from NIRCam Development Contract NAS50210 from NASA Goddard Space Flight Center to the University of Arizona. S.T. acknowledges support from the ERC Consolidator Grant funding scheme (project ConTExt, grant No.648179), FV from the Carlsberg Foundation Research Grant CF18-0388, and GEM from the Villum Fonden research grant 13160. The Cosmic Dawn Center is funded by the Danish National Research Foundation under grant No. 140. CP is supported by the Canadian Space Agency under a contract with NRC Herzberg Astronomy and Astrophysics. MA acknowledges support by NASA under award No 80NSSC19K1418. JSS is supported by NASA Hubble Fellowship grant \#HF2- 51446 awarded by the Space Telescope Science Institute, which is operated by the Association of Universities for Research in Astronomy, Inc., for NASA, under contract NAS5-26555. AM is supported by a Dunlap Fellowship at the Dunlap Institute for Astronomy \& Astrophysics, funded through an endowment established by the David Dunlap family and the University of Toronto.

Author Contributions: KEW proposed and carried out the observations, conducted the analysis in this paper, and authored the majority of the text. CCW performed the weighted stack of the data, helped to create Figure 3, and edited the text in the main body. LM performed the direct analysis of the ALMA flux densities and created the color images in Figure 1. JSS carried out the reduction and direct analysis of the raw ALMA data. MA reduced the HST images. GEM, AP, ST, and FV helped interpret the millimeter data and contributed to the dust and gas mass analysis. DN helped interpret the data in the context of cosmological simulation models. All authors, including RB, GBB, JL, AM, EJN, CP, KS, and PvD, contributed to the overall interpretation of the results and various aspects of the analysis and writing.

Author Information: Please send correspondence to Katherine E. Whitaker, kwhitaker@astro.umass.edu. 
The authors have no competing financial interests.

\section{References}

1. Muzzin, A., et al., "The Evolution of the Stellar Mass Functions of Star-forming and Quiescent Galaxies to $z=4$ from the COSMOS/UltraVISTA Survey", Astrophysical Journal, 777, 18 (2013).

2. Tacconi, L., et al. "High molecular gas fractions in normal massive star-forming galaxies in the young Universe", Nature, 463, 781 (2010)

3. Genzel, R., et al., "Combined CO and Dust Scaling Relations of Depletion Time and Molecular Gas Fractions with Cosmic Time, Specific Star-formation Rate, and Stellar Mass", Astrophysical Journal, 800, 20 (2015).

4. Tacconi, L., et al., "PHIBSS: Unified Scaling Relations of Gas Depletion Time and Molecular Gas Fractions", Astrophysical Journal, 853, 179 (2018).

5. Keres, D., et al., "How do galaxies get their gas?, Monthly Notices of the Royal Astronomical Society, 363,2 (2005).

6. Dekel, A., et al. "Cold streams in early massive hot haloes as the main mode of galaxy formation", Nature, 457, 451 (2009).

7. Ebeling, H., et al., "Thirty-fold: Extreme Gravitational Lensing of a Quiescent Galaxy at z=1.6", Astrophysical Journal, 852, 7 (2018).

8. Newman, N., et al., "Resolving Quiescent Galaxies at z>2. I. Search for Gravitationally Lensed Sources and Characterization of Their Structure, Stellar Populations, and Line Emission", Astrophysical Journal, 862, 125 (2018).

9. Toft, S., et al., "A massive, dead disk galaxy in the early Universe", Nature, 546, 510 (2017).

10. Man, A., et al., "An exquisitely deep view of quenching galaxies through the gravitational lens: Stellar population, morphology, and ionized gas", submitted to Astrophysical Journal September 2020.

11. Sargent, M. et al., "A Direct Constraint on the Gas Content of a Massive, Passively Evolving Elliptical Galaxy at $z=1.43$ ", Astrophysical Journal, 806, 20 (2015).

12. Gobat, R., et al., "The unexpectedly large dust and gas content of quiescent galaxies at $z>1.4$ ", Nature Astronomy, 2, 239 (2018).

13. Bezanson, R., et al., "Extremely Low Molecular Gas Content in a Compact, Quiescent Galaxy at z = 1.522", Astrophysical Journal, 873, 19 (2019).

14. Zavala, J., et al., "On the Gas Content, Star Formation Efficiency, and Environmental Quenching of Massive Galaxies in Protoclusters at z 2.0-2.5”, Astrophysical Journal, 887, 183 (2019).

15. Suess, K., et al., "Massive Quenched Galaxies at z 0.7 Retain Large Molecular Gas Reservoirs", Astrophysical Journal, 846, 14 (2017).

16. Spilker, J., et al., "Molecular Gas Contents and Scaling Relations for Massive, Passive Galaxies at Intermediate Redshifts from the LEGA-C Survey”, Astrophysical Journal, 860, 103 (2018). 
17. Scoville, N., et al., "ISM Masses and the Star formation Law at $Z=1$ to 6: ALMA Observations of Dust Continuum in 145 Galaxies in the COSMOS Survey Field", Astrophysical Journal, 820, 83 (2016).

18. Tadaki, K., et al., "Bulge-forming Galaxies with an Extended Rotating Disk at z 2", Astrophysical Journal, 824, 175 (2017).

19. Saintonge, A., et al., "xCOLD GASS: The Complete IRAM 30 m Legacy Survey of Molecular Gas for Galaxy Evolution Studies", Astrophysical Journal Supplement, 233, 22 (2017).

20. Li, Z., et al., "The Evolution of the Interstellar Medium in Post-starburst Galaxies", Astrophysical Journal, 879, 131 (2019).

21. Valentino, F., et al., "Quiescent Galaxies 1.5 Billion Years after the Big Bang and Their Progenitors", Astrophysical Journal, 889, 93 (2020).

22. Lagos, C., et al., "The origin of the atomic and molecular gas contents of early-type galaxies - II. Misaligned gas accretion", Monthly Notices of the Royal Astronomical Society, 448, 1271 (2015).

23. Dave, R., et al., "SIMBA: Cosmological simulations with black hole growth and feedback", Monthly Notices of the Royal Astronomical Society, 486, 2827 (2019).

24. Whitaker, K., et al., "Constraining the Low-mass Slope of the Star Formation Sequence at $0.5<z<$ 2.5”, Astrophysical Journal, 775, 104 (2014).

25. Ciotti, L., et al., "Radiative Feedback from Massive Black Holes in Elliptical Galaxies: AGN Flaring and Central Starburst Fueled by Recycled Gas", Astrophysical Journal, 665, 1038 (2007).

26. Akhshik, M., et al., "Recent Star-formation in a Massive Slowly-Quenched Lensed Quiescent Galaxy at $z=1.88$ ", submitted to Astrophysical Journal Letters October 2020.

27. Dekel, A. \& Birnboim, Y., "Galaxy bimodality due to cold flows and shock heating", Monthly Notices of the Royal Astronomical Society, 368, 1 (2006).

28. Cheung, E., et al., "Suppressing star formation in quiescent galaxies with supermassive black hole winds", Nature, 533, 504 (2016).

29. Whitaker, K., et al., "Quiescent Galaxies in the 3D-HST Survey: Spectroscopic Confirmation of a Large Number of Galaxies with Relatively Old Stellar Populations at z 2" Astrophysical Journal Letters, 770, 39 (2013).

30. Johansson, P., et al., "Gravitational Heating Helps Make Massive Galaxies Red and Dead", Astrophysical Journal Letters, 697, 38 (2009).

31. Chabrier, G., "Galactic Stellar and Substellar Initial Mass Function", Publications of the Astronomical Society of the Pacific, 115, 763 (2003).

32. Akhshik, M., et al., "REQUIEM-2D Methodology: Spatially Resolved Stellar Populations of Massive Lensed Quiescent Galaxies from Hubble Space Telescope 2D Grism Spectroscopy", Astrophysical Journal, 900, 184 (2020).

33. Calzetti, D., et al., "The Dust Content and Opacity of Actively Star-forming Galaxies", Astrophysical Journal, 533, 682 (2000). 
34. Lee, B., et al., "The Intrinsic Characteristics of Galaxies on the SFR-M* Plane at $1.2<z<4$ : I. The Correlation between Stellar Age, Central Density, and Position Relative to the Main Sequence", Astrophysical Journal, 853, 131 (2018).

35. Carvajal, R., et al., "The ALMA Frontier Fields Survey. V. ALMA Stacking of Lyman-Break Galaxies in Abell 2744, Abell 370, Abell S1063, MACSJ0416.1-2403 and MACSJ1149.5+2223", Astronomy \& Astrophysics, 633, 160 (2020).

36. Greve, T., et al., "Submillimeter Observations of Millimeter Bright Galaxies Discovered by the South Pole Telescope”, Astrophysical Journal, 756, 101 (2012).

37. Scoville, N., et al., "The Evolution of Interstellar Medium Mass Probed by Dust Emission: ALMA Observations at z = 0.3-2", Astrophysical Journal, 783, 84 (2014)

38. Zhang, C., et al., "Nearly all Massive Quiescent Disk Galaxies Have a Surprisingly Large Atomic Gas Reservoir", Astrophysical Journal Letters, 884, 52 (2019).

39. Sage, L., et al., "The Cool ISM in Elliptical Galaxies. I. A Survey of Molecular Gas”, Astrophysical Journal, 657, 232 (2007).

40. Li, Q., et al., "The dust-to-gas and dust-to-metal ratio in galaxies from z $=0$ to 6", Monthly Notices of the Royal Astronomical Society, 490, 1425 (2019).

41. Smercina, A., et al., "After the Fall: The Dust and Gas in E+A Post-starburst Galaxies", Astrophysical Journal, 855, 51 (2018).

42. Williams, C., et al., "ALMA measures rapidly depleted molecular gas reservoirs in massive quiescent galaxies at $z \sim 1.5 "$, accepted for publication in Astrophysical Journal November 2020.

43. Man, A., et al., "Low molecular gas fraction in massive quenched galaxies at z>=2" (in prep)

44. Smith, M., et al., "The Herschel Reference Survey: Dust in Early-type Galaxies and across the Hubble Sequence", Astrophysical Journal, 748, 123 (2012).

45. Magdis, G., et al., “ The Interstellar Medium of Quiescent Galaxies and its Evolution With Time”, submitted to Astronomy \& Astrophysics in September 2020.

46. Saintonge, A., et al., "Validation of the Equilibrium Model for Galaxy Evolution to z 3 through Molecular Gas and Dust Observations of Lensed Star-forming Galaxies", Astrophysical Journal, 778, 2 (2013).

47. Franco, M., et al., "GOODS-ALMA: The slow downfall of star formation in z = 2-3 massive galaxies”, Astronomy \& Astrophysics, 643, 30 (2020).

48. Tacconi, L., et al., "Submillimeter Galaxies at z 2: Evidence for Major Mergers and Constraints on Lifetimes, IMF, and CO- $\mathrm{H}_{2}$ Conversion Factor", Astrophysical Journal, 680, 246 (2008).

49. Daddi, E., et al., "Very High Gas Fractions and Extended Gas Reservoirs in z = 1.5 Disk Galaxies”, Astrophysical Journal, 713, 686 (2010).

50. Tacconi, L., et al. "High molecular gas fractions in normal massive star-forming galaxies in the young Universe”, Nature, 463, 781 (2010) 
51. Silverman, J., et al., "A Higher Efficiency of Converting Gas to Stars Pushes Galaxies at z 1.6 Well Above the Star-forming Main Sequence”, Astrophysical Journal Letters, 812, 23 (2015).

52. Decarli, R., et al., "The ALMA Spectroscopic Survey in the Hubble Ultra Deep Field: Molecular Gas Reservoirs in High-redshift Galaxies”, Astrophysical Journal, 833, 70 (2016).

53. Rudnick, G., et al. “Deep CO(1-0) Observations of z = 1.62 Cluster Galaxies with Substantial Molecular Gas Reservoirs and Normal Star Formation Efficiencies”, Astrophysical Journal, 849, 27 (2017).

54. Spilker, J., et al., “Low Gas Fractions Connect Compact Star-forming Galaxies to Their z 2 Quiescent Descendants", 832, 19 (2016).

55. Aravena, M., et al., "The ALMA Spectroscopic Survey in the Hubble Ultra Deep Field: The Nature of the Faintest Dusty Star-forming Galaxies”, Astrophysical Journal, 901, 79 (2020).

\section{Figures}

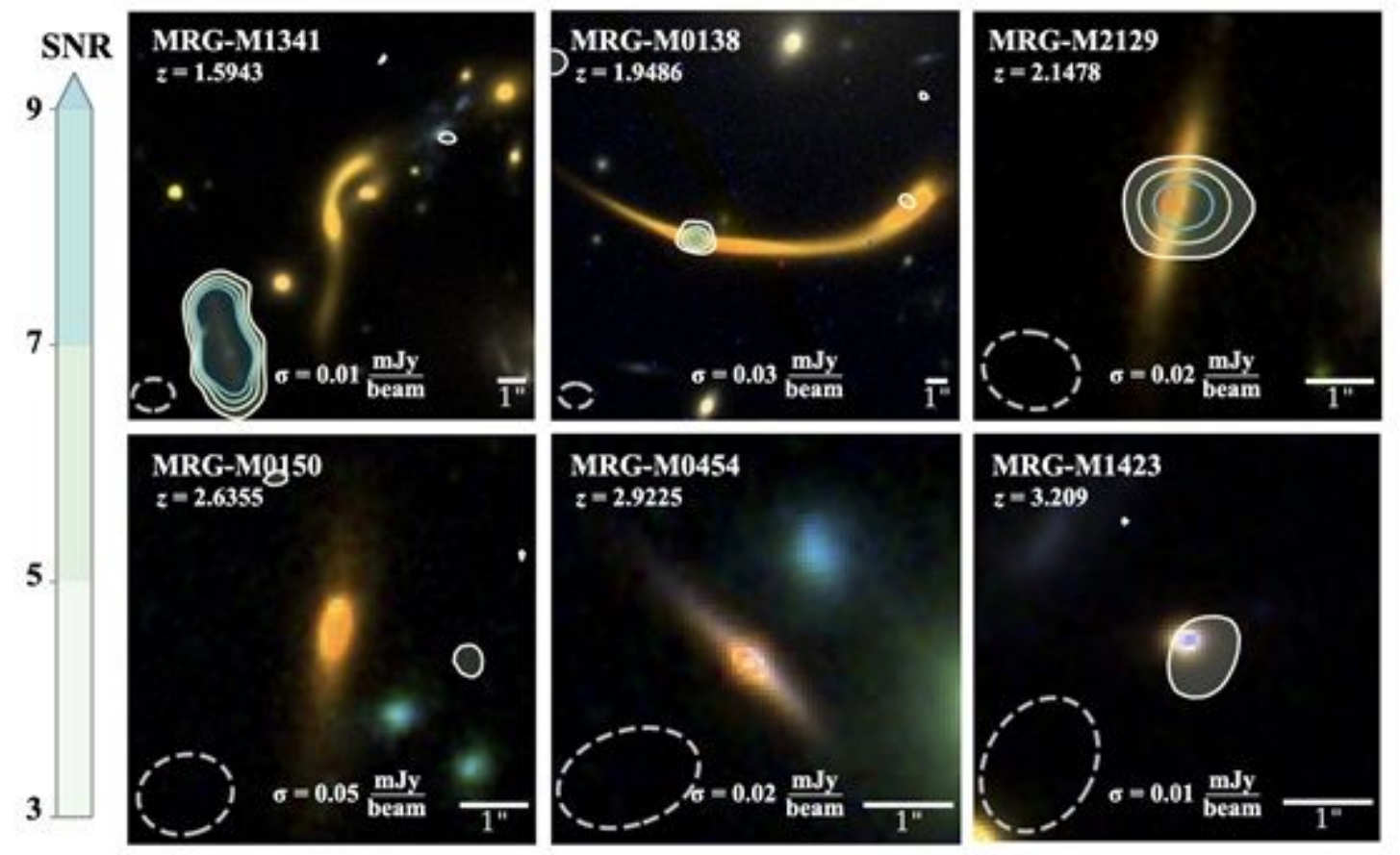

\section{Figure 1}

A sample of strong lensed massive quiescent galaxies, rank-ordered from $z=1.6$ to $z=3.2$, showing a composite HST color image (iF814W, JF125W, HF160W generally, substituting JF110W for MRG-M0454) and contours of ALMA/Band 6 dust continuum observations. Each image is centered on the target galaxy, whose redshift is listed in the upper-left corner. The dashed ellipse indicates the ALMA beam size. 

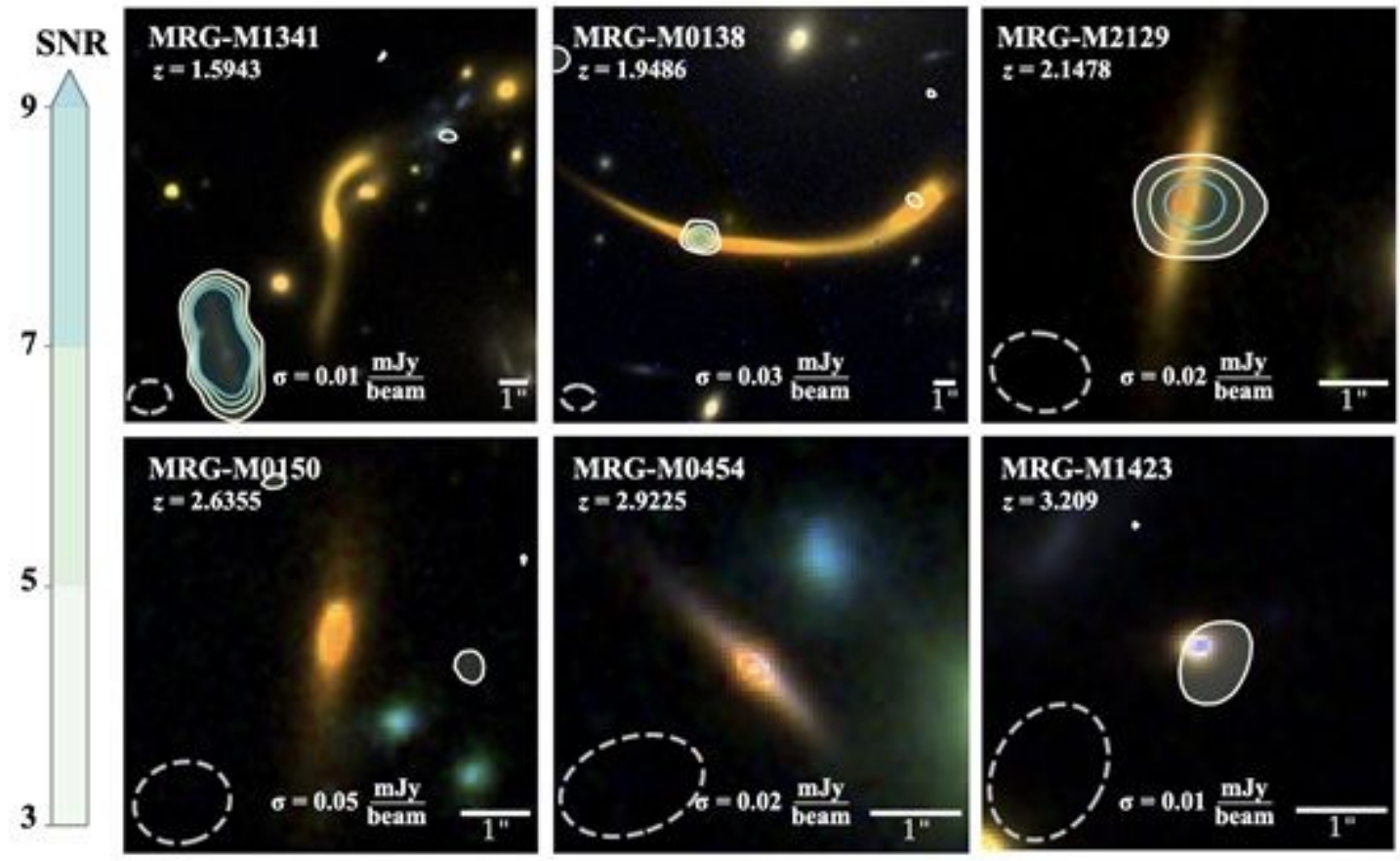

Figure 1

A sample of strong lensed massive quiescent galaxies, rank-ordered from $z=1.6$ to $z=3.2$, showing a composite HST color image (iF814W, JF125W, HF160W generally, substituting JF110W for MRG-M0454) and contours of ALMA/Band 6 dust continuum observations. Each image is centered on the target galaxy, whose redshift is listed in the upper-left corner. The dashed ellipse indicates the ALMA beam size.

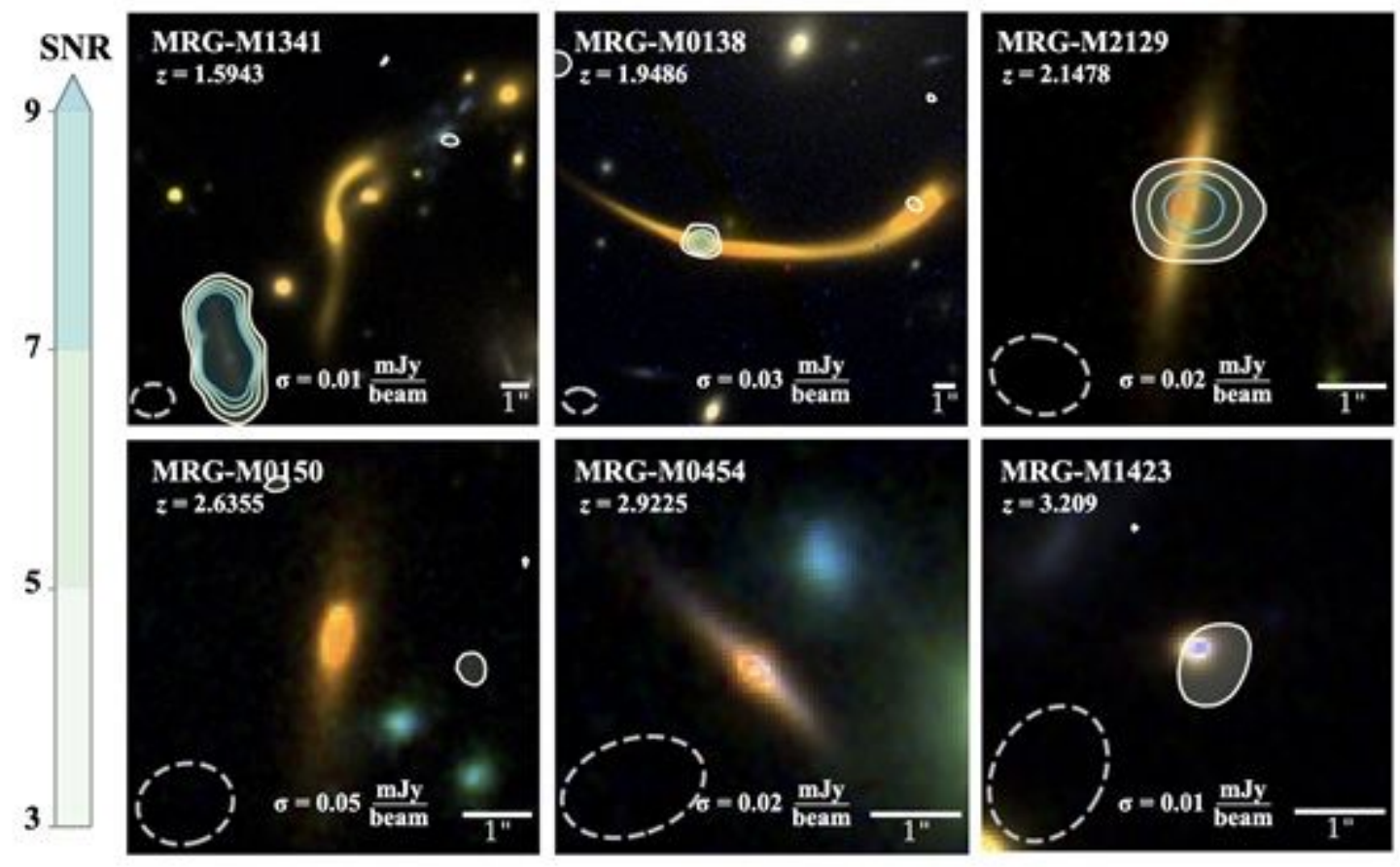

Figure 1 
A sample of strong lensed massive quiescent galaxies, rank-ordered from $z=1.6$ to $z=3.2$, showing a composite HST color image (iF814W, JF125W, HF160W generally, substituting JF110W for MRG-M0454) and contours of ALMA/Band 6 dust continuum observations. Each image is centered on the target galaxy, whose redshift is listed in the upper-left corner. The dashed ellipse indicates the ALMA beam size.

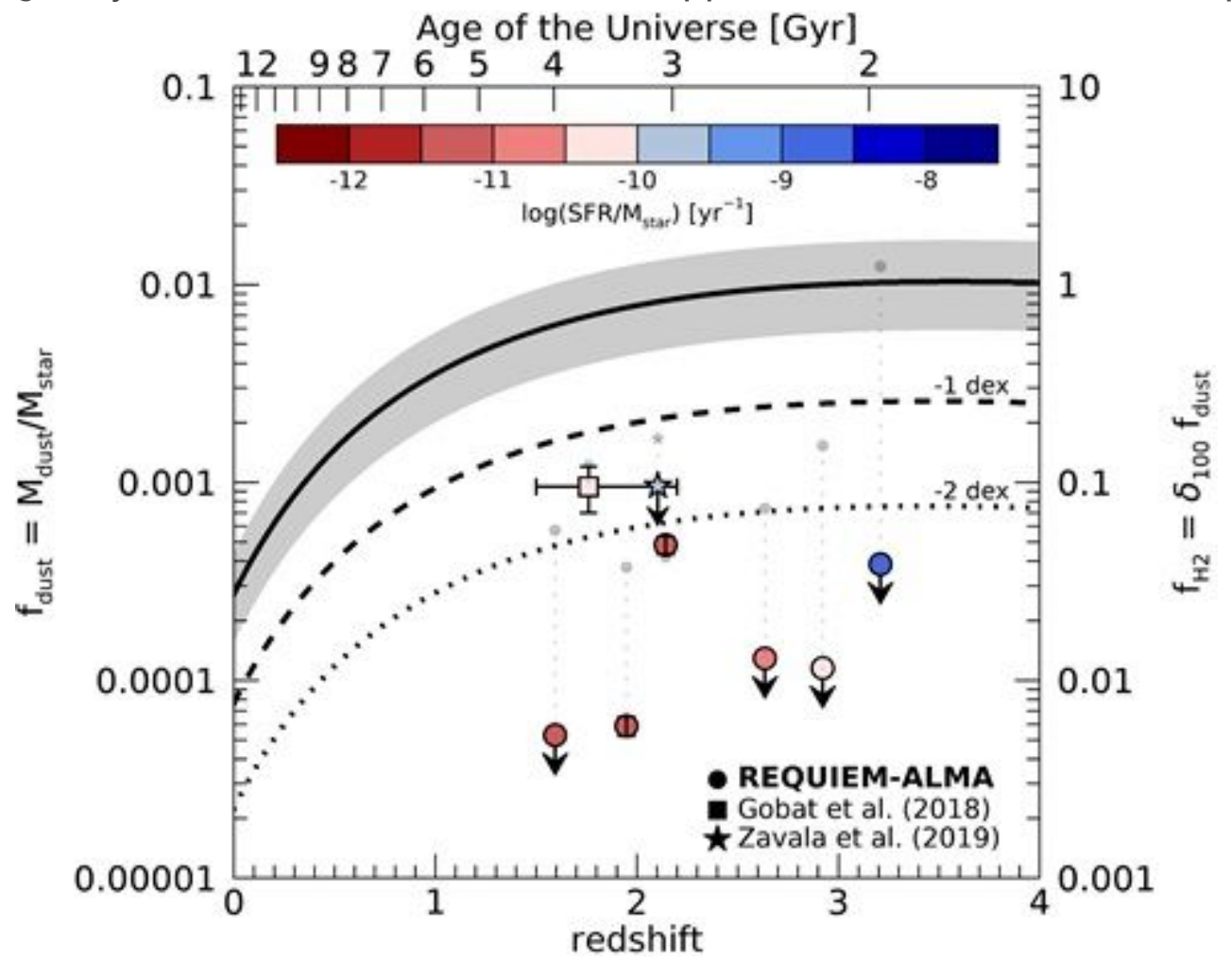

Figure 2

Measurements of fdust for distant lensed quiescent galaxies (circles) are extremely low given their starformation rate per unit stellar mass (SSFR). We compare existing measurements in the literature of quiescent galaxies (see Methods), using identical conversions herein to our sample. The transparent symbols represent the predicted $\mathrm{fH} 2$ from empirical scaling relations4 given sSFR. The inferred $\mathrm{fH} 2$ (right axis) and scaling relations for $\log (\mathrm{M} \mathbb{\mathrm { M }} / \mathrm{M} \odot)=11$ on the average $\log (\mathrm{SFR}) \log (\mathrm{M} \mathrm{Z})$ relation (solid), $1 \mathrm{dex}$ (dashed), and 2 dex below (dotted) assume a molecular gas to dust mass ratio of 100 . The shaded region shows the upper bound set by the lowest stellar mass in our sample $(\log (M \otimes / M \odot)=10.4)$, and vice versa for the highest stellar mass $(\log (\mathrm{M} \otimes / \mathrm{M} \odot)=11.7)$. 


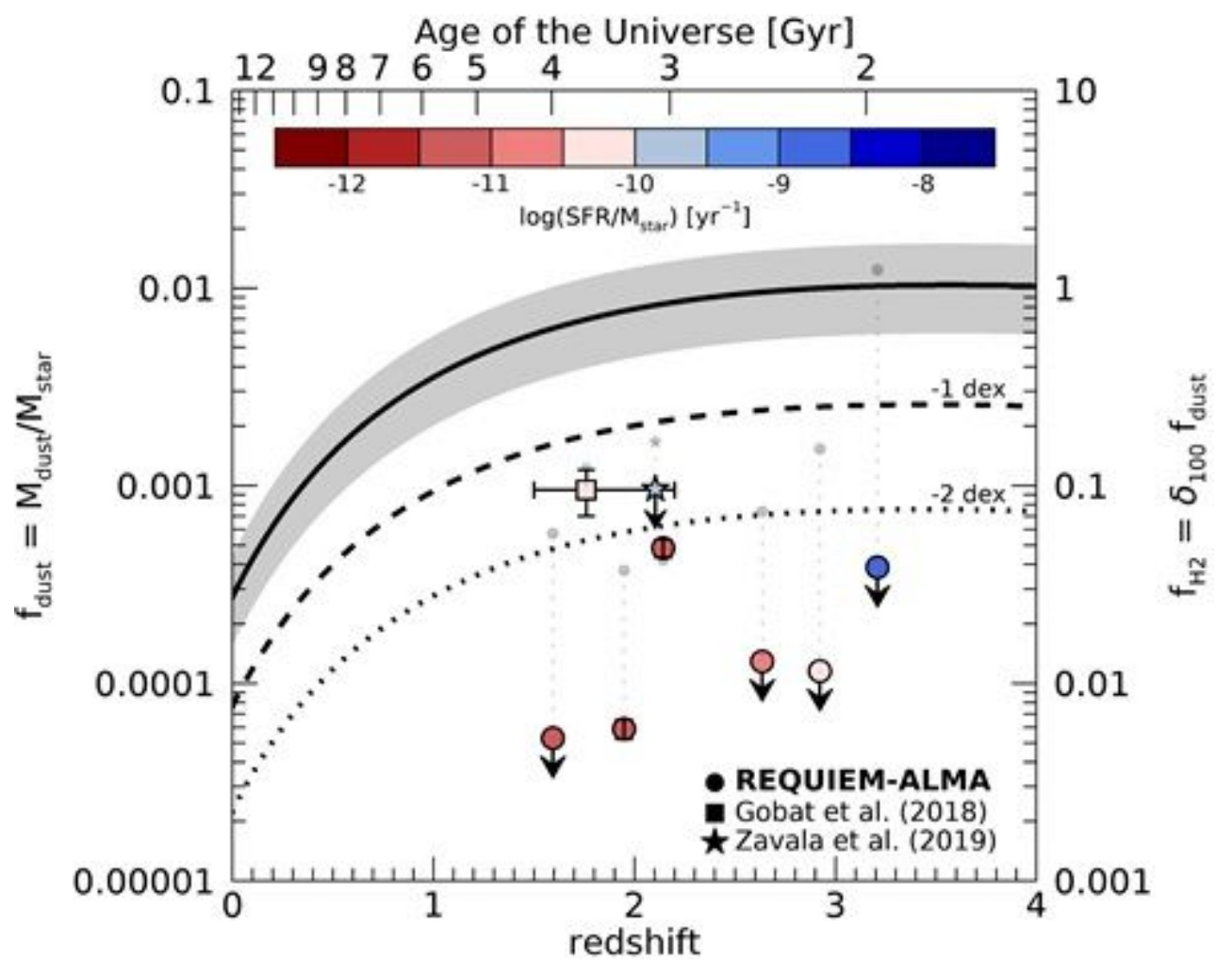

Figure 2

Measurements of fdust for distant lensed quiescent galaxies (circles) are extremely low given their starformation rate per unit stellar mass (SSFR). We compare existing measurements in the literature of quiescent galaxies (see Methods), using identical conversions herein to our sample. The transparent symbols represent the predicted $\mathrm{fH} 2$ from empirical scaling relations4 given sSFR. The inferred $\mathrm{fH} 2$ (right axis) and scaling relations for $\log (\mathrm{M} \nabla / \mathrm{M} \odot)=11$ on the average $\log (\mathrm{SFR}) \log (\mathrm{M} \nabla)$ relation (solid), 1 dex (dashed), and 2 dex below (dotted) assume a molecular gas to dust mass ratio of 100 . The shaded region shows the upper bound set by the lowest stellar mass in our sample $(\log (M \otimes / M \odot)=10.4)$, and vice versa for the highest stellar mass $(\log (M \otimes / M \odot)=11.7)$. 


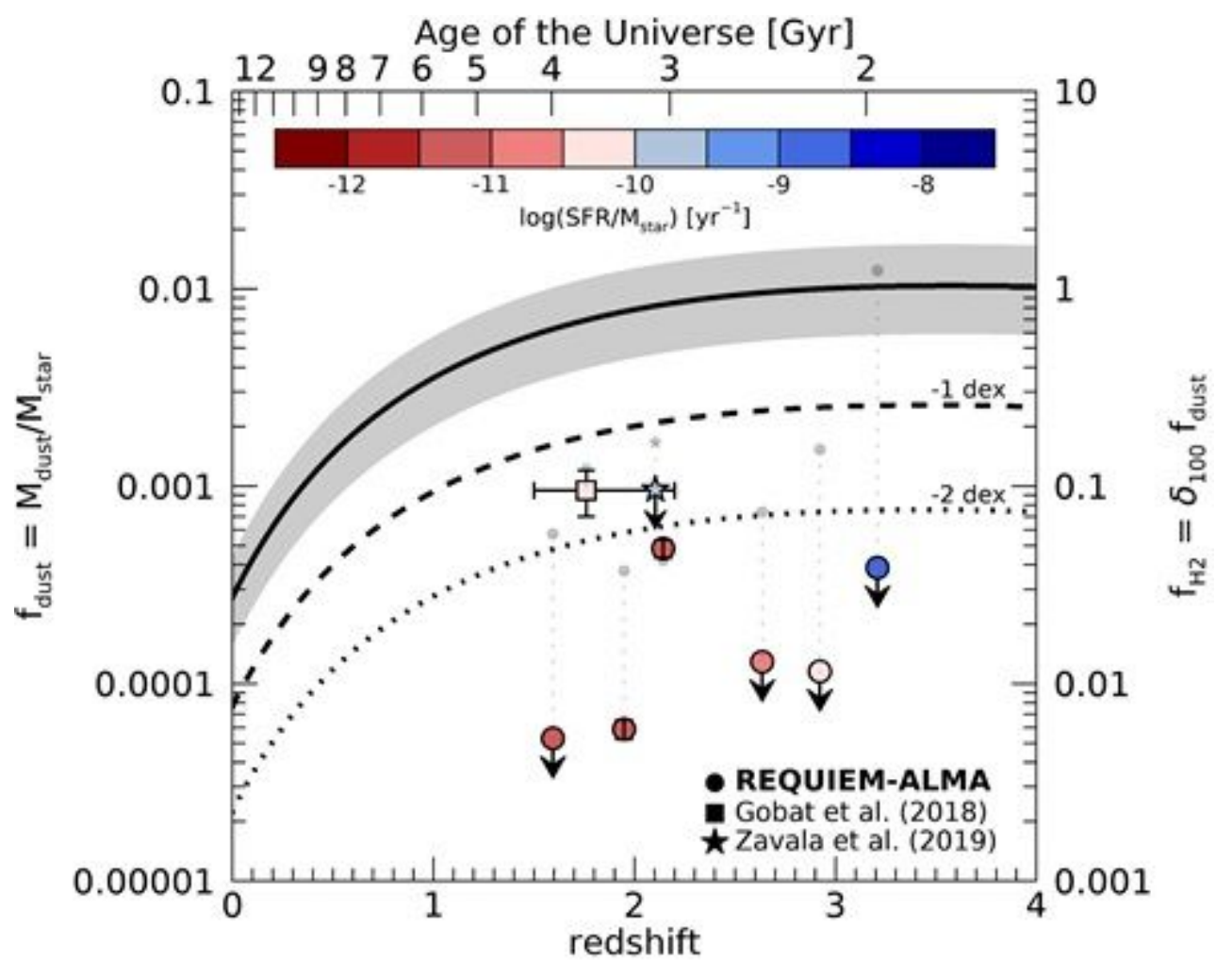

Figure 2

Measurements of fdust for distant lensed quiescent galaxies (circles) are extremely low given their starformation rate per unit stellar mass (SSFR). We compare existing measurements in the literature of quiescent galaxies (see Methods), using identical conversions herein to our sample. The transparent symbols represent the predicted $\mathrm{fH} 2$ from empirical scaling relations4 given sSFR. The inferred $\mathrm{fH} 2$ (right axis) and scaling relations for $\log (\mathrm{M} \nabla / \mathrm{M} \odot)=11$ on the average $\log (\mathrm{SFR}) \log (\mathrm{M} \nabla)$ relation (solid), 1 dex (dashed), and 2 dex below (dotted) assume a molecular gas to dust mass ratio of 100 . The shaded region shows the upper bound set by the lowest stellar mass in our sample $(\log (M \otimes / M \odot)=10.4)$, and vice versa for the highest stellar mass $(\log (\mathrm{M} \otimes / \mathrm{M} \odot)=11.7)$. 


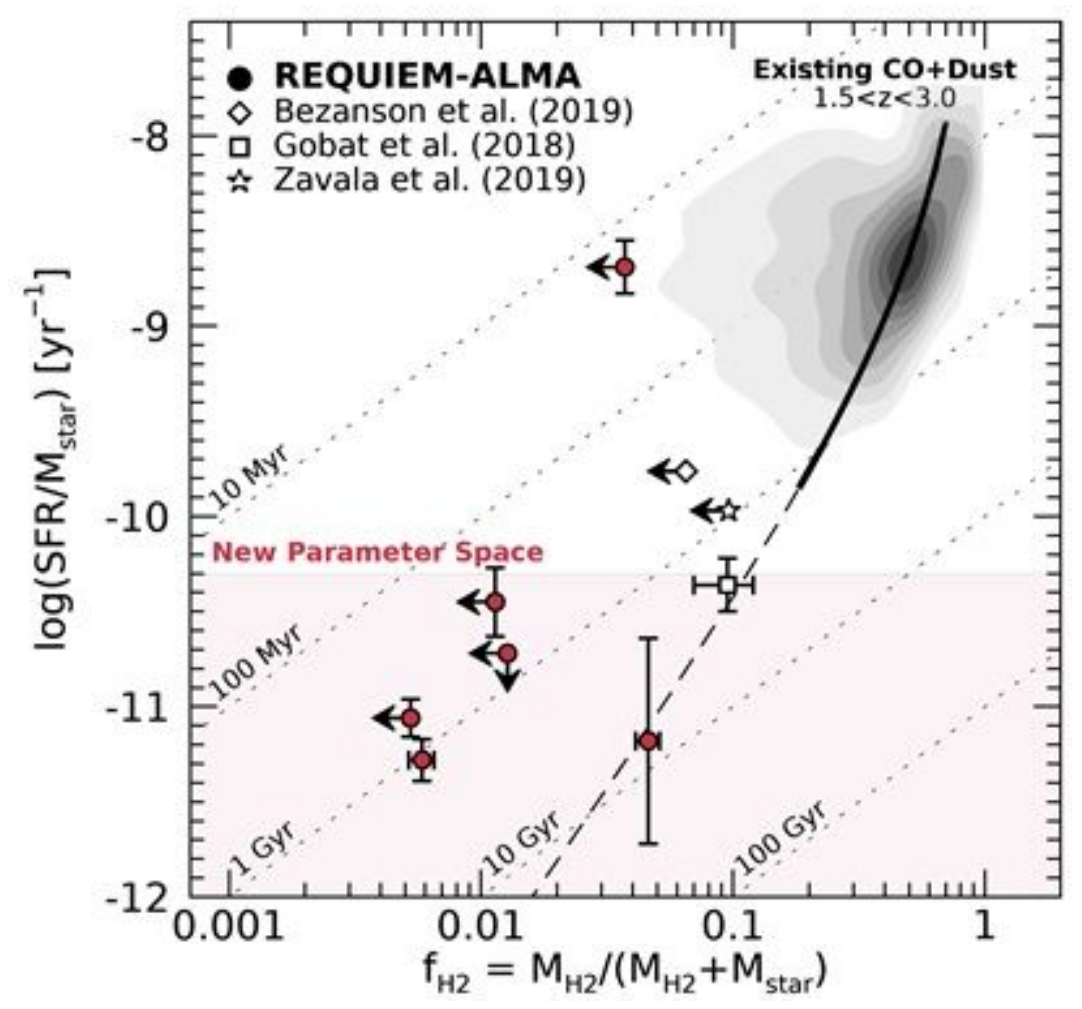

Figure 3

The molecular gas fraction $\mathrm{fH} 2$ is significantly lower at a given star-formation rate per stellar mass (SSFR $\equiv S F R / M \rrbracket)$ for distant lensed quiescent galaxies at $z>1.5$ when compared to the compilation of existing $\mathrm{CO}$ and dust measurements of similarly-massive star-forming galaxies (contours, see Methods). Our sample explores an order of magnitude lower SSFR and higher redshifts, finding median molecular gas fractions a factor of 10 lower than existing measurements for distant quiescent galaxies (see Methods). The data are consistent with rapid gas depletion, on average following the tracks for constant gas depletion timescales ranging of order $\sim 1$ billion years (dotted lines). 


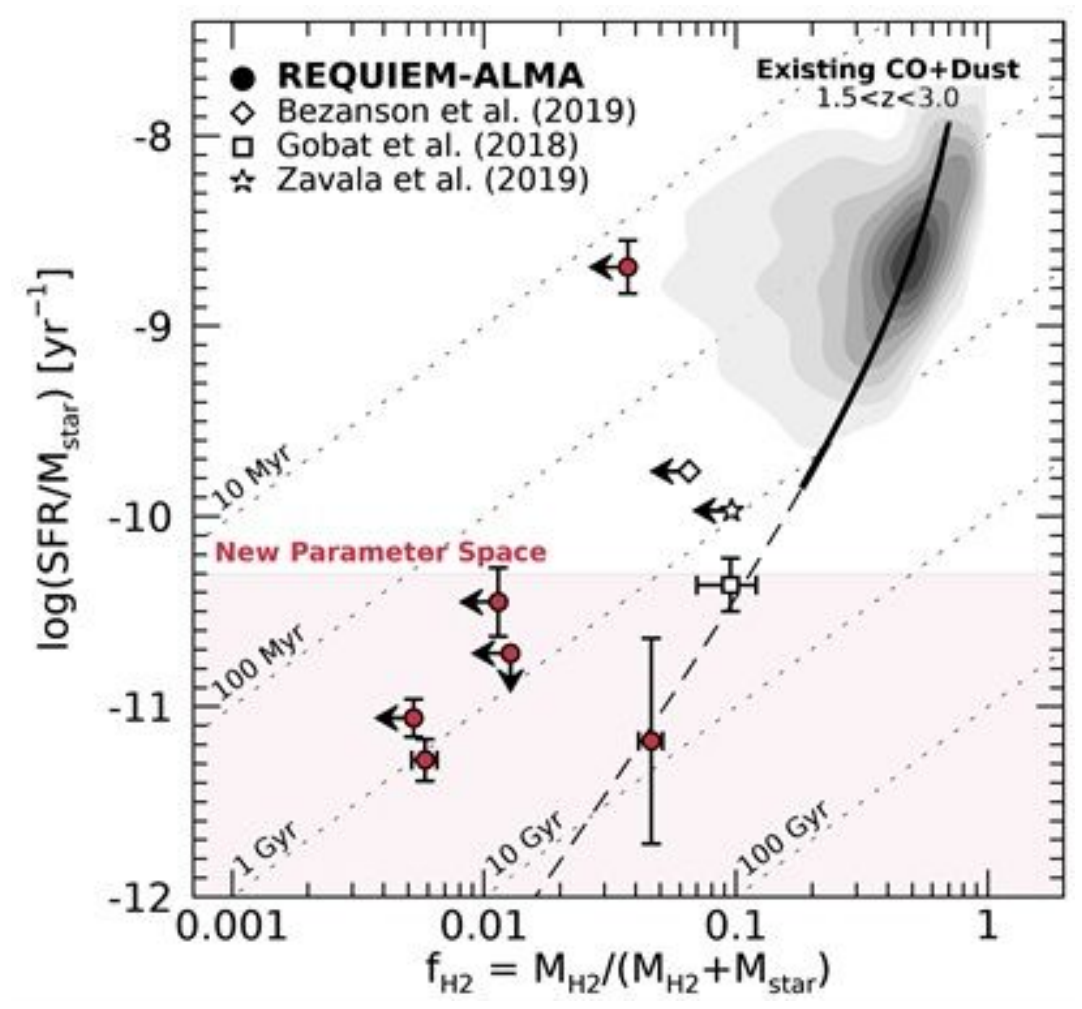

Figure 3

The molecular gas fraction $\mathrm{fH} 2$ is significantly lower at a given star-formation rate per stellar mass (SSFR $\equiv S F R / M \rrbracket)$ for distant lensed quiescent galaxies at $z>1.5$ when compared to the compilation of existing $\mathrm{CO}$ and dust measurements of similarly-massive star-forming galaxies (contours, see Methods). Our sample explores an order of magnitude lower SSFR and higher redshifts, finding median molecular gas fractions a factor of 10 lower than existing measurements for distant quiescent galaxies (see Methods). The data are consistent with rapid gas depletion, on average following the tracks for constant gas depletion timescales ranging of order $\sim 1$ billion years (dotted lines). 


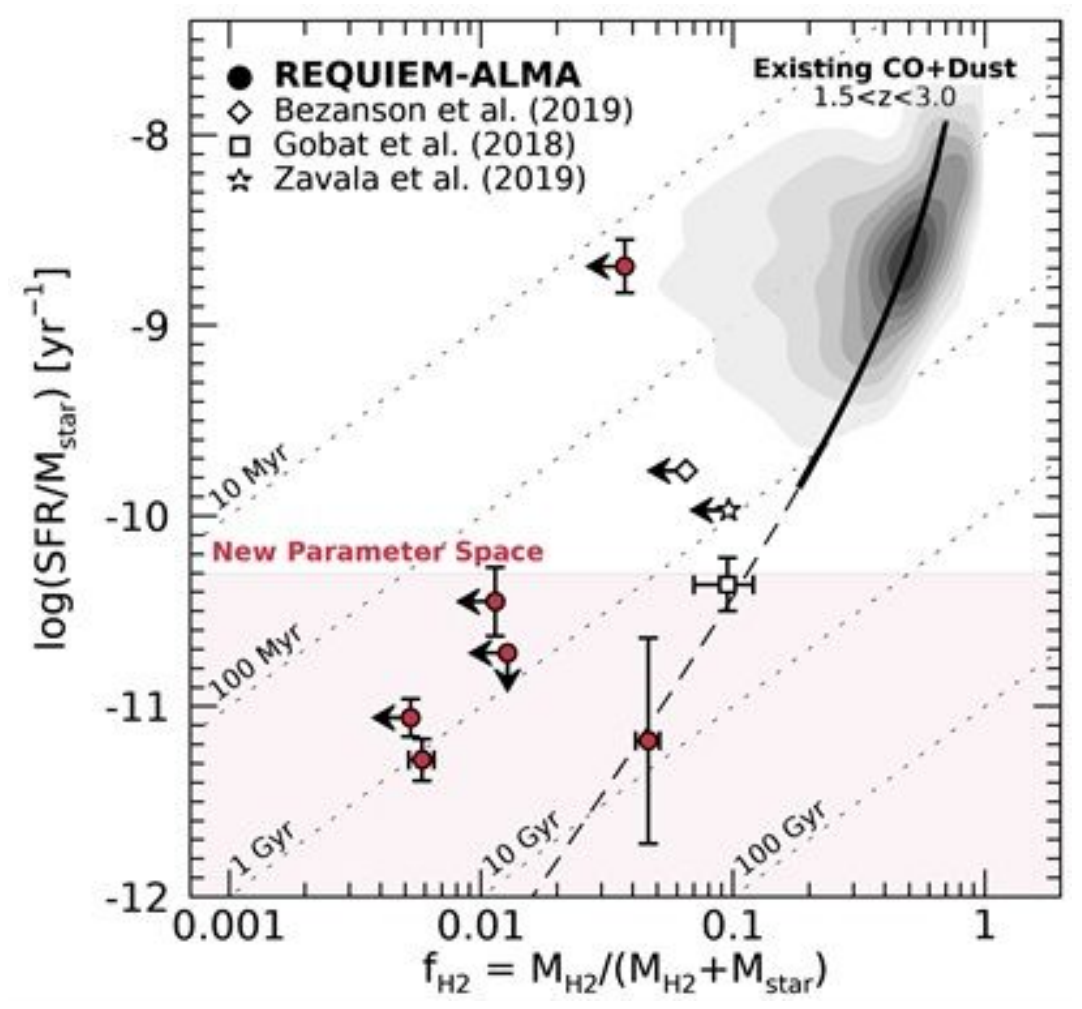

Figure 3

The molecular gas fraction $\mathrm{fH} 2$ is significantly lower at a given star-formation rate per stellar mass (SSFR $\equiv S F R / M \rrbracket)$ for distant lensed quiescent galaxies at $z>1.5$ when compared to the compilation of existing $\mathrm{CO}$ and dust measurements of similarly-massive star-forming galaxies (contours, see Methods). Our sample explores an order of magnitude lower SSFR and higher redshifts, finding median molecular gas fractions a factor of 10 lower than existing measurements for distant quiescent galaxies (see Methods). The data are consistent with rapid gas depletion, on average following the tracks for constant gas depletion timescales ranging of order $\sim 1$ billion years (dotted lines). 